\title{
Desynchronization of chaos in coupled logistic maps
}

\author{
Maistrenko, Yu.; Maistrenko, V. L.; Popovych, O.; Mosekilde, Erik
}

Published in:

Physical Review E. Statistical, Nonlinear, and Soft Matter Physics

Link to article, DOI:

10.1103/PhysRevE.60.2817

Publication date:

1999

Document Version

Publisher's PDF, also known as Version of record

Link back to DTU Orbit

Citation (APA):

Maistrenko, Y., Maistrenko, V. L., Popovych, O., \& Mosekilde, E. (1999). Desynchronization of chaos in coupled logistic maps. Physical Review E. Statistical, Nonlinear, and Soft Matter Physics, 60(3), 2817-2830. https://doi.org/10.1103/PhysRevE.60.2817

\section{General rights}

Copyright and moral rights for the publications made accessible in the public portal are retained by the authors and/or other copyright owners and it is a condition of accessing publications that users recognise and abide by the legal requirements associated with these rights.

- Users may download and print one copy of any publication from the public portal for the purpose of private study or research.

- You may not further distribute the material or use it for any profit-making activity or commercial gain

- You may freely distribute the URL identifying the publication in the public portal 


\title{
Desynchronization of chaos in coupled logistic maps
}

\author{
Yu. L. Maistrenko, ${ }^{1}$ V. L. Maistrenko, ${ }^{1}$ O. Popovych, ${ }^{1}$ and E. Mosekilde ${ }^{2}$ \\ ${ }^{1}$ Institute of Mathematics, National Academy of Sciences, Kiev, 252601, Ukraine \\ ${ }^{2}$ Department of Physics, Technical University of Denmark, 2800 Lyngby, Denmark
}

(Received 22 January 1999)

\begin{abstract}
When identical chaotic oscillators interact, a state of complete or partial synchronization may be attained in which the motion is restricted to an invariant manifold of lower dimension than the full phase space. Riddling of the basin of attraction arises when particular orbits embedded in the synchronized chaotic state become transversely unstable while the state remains attracting on the average. Considering a system of two coupled logistic maps, we show that the transition to riddling will be soft or hard, depending on whether the first orbit to lose its transverse stability undergoes a supercritical or subcritical bifurcation. A subcritical bifurcation can lead directly to global riddling of the basin of attraction for the synchronized chaotic state. A supercritical bifurcation, on the other hand, is associated with the formation of a so-called mixed absorbing area that stretches along the synchronized chaotic state, and from which trajectories cannot escape. This gives rise to locally riddled basins of attraction. We present three different scenarios for the onset of riddling and for the subsequent transformations of the basins of attraction. Each scenario is described by following the type and location of the relevant asynchronous cycles, and determining their stable and unstable invariant manifolds. One scenario involves a contact bifurcation between the boundary of the basin of attraction and the absorbing area. Another scenario involves a long and interesting series of bifurcations starting with the stabilization of the asynchronous cycle produced in the riddling bifurcation and ending in a boundary crisis where the stability of an asynchronous chaotic state is destroyed. Finally, a phase diagram is presented to illustrate the parameter values at which the various transitions occur. [S1063-651X(99)04509-2]
\end{abstract}

PACS number(s): 05.45.-a

\section{INTRODUCTION}

Interacting chaotic oscillators are of interest in connection with a wide range of problems in science and technology [1]. In the biological sciences, for instance, one of the fundamental problems is to understand how a group of cells or functional units, each displaying complicated nonlinear dynamics, can interact with one another to produce different forms of coordinated function at a higher organizational level [2].

Studies performed by a number of investigators have disclosed how chaotic interaction can lead to a variety of different synchronization phenomena. In phase synchronization $[3,4]$, for instance, the interacting chaotic systems adjust their phases such that the mean return times to some Poincaré secant are related in a rational manner. The amplitudes, on the other hand, can vary quite differently. Full synchronization, in which both the phases and amplitudes develop in precisely the same way, can be achieved through the coupling of two (or more) identical oscillators [5]. In the presence of a parameter mismatch between the chaotic systems, lag synchronization may be observed [6]. Here the amplitudes of the two subsystems are correlated, but there is a phase shift between their motions. Finally, if more than two oscillators are involved, one may observe the phenomenon of clustering [7] or partial synchronization [8], where some of the oscillators synchronize and others do not. This is often associated with the coexistence of a number of different synchronized states, each with its own basin of attraction.

Full synchronization is of interest in connection, for instance, with the development of new types of communication techniques that exploit the possibility of masking a message by mixing it with a chaotic signal [9]. With both the amplitudes and phases of the interacting oscillators varying in the same way, the synchronized chaotic state will be restricted to a smooth invariant manifold of lower dimension than the full phase space. A similar situation can also arise in nonlinear dynamic systems with built-in symmetries [10,11]. A main problem is then related to the stability of the synchronized state to perturbations transverse to the synchronization manifold. Another important question concerns what happens when the synchronization breaks down. Recent studies of these and related problems have lead to the discovery of a variety of new phenomena, including riddled basins of attraction [10,11], attractor bubbling [12], and on-off intermittency $[13,14]$.

Riddled basins of attraction may be observed in regions of parameter space where the synchronized chaotic state is attracting on the average (the typical transverse Lyapunov exponents are negative), while at the same time particular orbits embedded in the chaotic set are transversely unstable (the corresponding eigenvalues are numerically larger that one) $[11,12]$. The basin of attraction for the synchronized chaotic state may then become a fat fractal, riddled with initial conditions from which the trajectories diverge toward infinity or approach other asymptotic states. The transition in which the first orbit on the chaotic set becomes transversely unstable is referred to as the riddling bifurcation. For a system of two coupled one-dimensional maps, this bifurcation may be either a pitchfork bifurcation (eigenvalue +1 ) [15] or a period-doubling bifurcation (eigenvalue -1) [16].

However, transverse destabilization of orbits embedded in the chaotic set is not sufficient for an observable riddling to arise. This will depend on the global dynamics of the system. Having left the locally repelling regions in the neighborhood of the chaotic set, the trajectories may wander around in phase space without ever approaching another attractor (or 
escaping to infinity). Sooner or later most of them will return to the neighborhood of the synchronization manifold. Some may again be mapped into repelling tongues, while others will be attracted by the chaotic set, and at the end almost all trajectories starting close to the invariant manifold will end up in the synchronized state. This produces the phenomenon that has been called local riddling $[12,16,17]$. In the presence of noise, a locally riddled basin of attraction will manifest itself in the form of attractor bubbling [12,18], where intervals of desynchronized bursting behavior occur.

Denoting the synchronized chaotic state by $A$, its $\delta$ neighborhood by $U_{\delta}(A)$, and its basin of attraction by $\beta(A)$, the basin is said to be locally riddled if there exists a $\delta$ such that $A$ attracts almost all points from $U_{\delta}(A)$, i.e., $\mu\left\{\beta(A) \cap U_{\delta}(A)\right\}=\mu\left\{U_{\delta}(A)\right\}$, where $\mu\{\cdot\}$ denotes a Lebesgue measure. In contrast to the case of asymptotic stability, however, the transverse repulsive character of orbits embedded in the synchronized chaotic set implies that the neighborhood of any point of $A$ will contain a positive measure set of points that leave $U_{\delta}(A)$ in a finite number of iterations.

Alternatively, the global dynamics of the system may be such that it allows direct access for trajectories repelled from the neighborhood of the transversely destabilized orbit (as well as from the neighborhoods of its dense set of preimages) to go to some other attractor (or infinity). This is the case of global riddling. $A$ then attracts a positive Lebesgue measure set of points from $U_{\delta}(A)$, but not the full measure, i.e., $0<\mu\left\{\beta(A) \cap U_{\delta}(A)\right\}<\mu\left\{U_{\delta}(A)\right\}$. We have previously described $[16,17]$ how the distinction between these two types of riddling for a system of two coupled, noninvertible maps depends on the existence of a so-called absorbing area $[19,20]$ that controls the global dynamics of the system, and can restrain trajectories starting near the synchronized chaotic set from reaching other limiting states.

The bubbling transition itself may be characterized as being either soft or hard. This distinction was introduced by Venkataramani et al. [18] to describe two different situations that can be observed immediately after the first orbit has lost its transverse stability. After a soft transition, trajectories starting in the neighborhood of the synchronization manifold will remain close to this manifold. After a hard transition, on the other hand, trajectories starting close to the synchronization manifold can immediately move far away in phase space, and some may approach other attractors. We have recently shown [21] how the distinction between a soft and a hard riddling bifurcation is related to the supercritical or subcritical nature of the transverse bifurcation in which the first orbit embedded in the synchronized chaotic set loses its stability. At the same time we have established general analytical conditions for the transverse bifurcation of a periodic orbit to be either supercritical or subcritical. This derivation was based on the construction of an asymptotical onedimensional map acting along the transverse invariant manifolds of the orbit that first loses its stability. In a supercritical transverse destabilization of a periodic orbit, the unstable manifolds of the asynchronous saddle cycle(s) born in the bifurcation (together with elements of the boundary of the absorbing area) will form a so-called mixed absorbing area that stretches along the synchronized chaotic set, and from which trajectories starting near the chaotic set cannot escape.
As the asynchronous saddle cycle(s) under variation of a control parameter move(s) away from the synchronization manifold, the width of the mixed absorbing area will grow. This leads to a synchronization error that increases as $\sqrt{\left|\varepsilon-\varepsilon_{c}\right|}$. Here $\left|\varepsilon-\varepsilon_{c}\right| \ll 1$ denotes the distance of the control parameter from the bifurcation point.

As opposed to the distinction between locally and globally riddled basins of attraction, the distinction between soft and hard riddling bifurcations only involves local conditions close to the synchronization manifold. A hard riddling bifurcation may lead to locally or globally riddled basins of attraction, depending on the conditions far from the synchronization manifold. As we shall show, however, immediately after a soft riddling bifurcation, the basin of attraction can be locally riddled only.

The purpose of the present paper is to illustrate these concepts in more detail by describing three different scenarios for the onset of riddling and for the subsequent development of the basin of attraction for a system of two coupled logistic maps. Each scenario is described by following the type and location of the relevant asynchronous cycles and determine their stable and unstable invariant manifolds. We also determine both the absorbing and mixed absorbing areas, and discuss their significance for the observed dynamics. The first scenario illustrates how the transition from locally to globally riddled basins of attraction can occur via a contact bifurcation between the basin of attraction for the synchronized chaotic state and its absorbing area $[19,21]$. The second scenario involves a direct transition to global riddling following a subcritical transverse bifurcation of a synchronized periodic orbit. In this case, the mixed absorbing area exists before the riddling bifurcation, and disappears in the moment of bifurcation.

The third scenario involves a long and interesting sequence of bifurcations following the destabilization of the synchronous period- 2 cycle in a supercritical transverse period doubling. In this case, the asynchronous period-4 saddle produced in the riddling bifurcation stabilizes in an inverse subcritical period-doubling bifurcation before the contact bifurcation between the basin of attraction and the absorbing area takes place. This gives rise to the emergence of a new attracting state inside the absorbing area for the synchronized chaotic set. Elements of this scenario were recently described by Bischi and Gardini [22]. Finally, we present a phase diagram delineating the regions in parameter space where the various solutions exist. This provides a clear picture of the conditions for the different scenarios to occur.

\section{CONDITIONS FOR SOFT AND HARD RIDDLING}

Let us consider the system

$$
F:\left\{\begin{array}{l}
x \\
y
\end{array}\right\} \rightarrow\left\{\begin{array}{l}
f_{a}(x)+\varepsilon(y-x) \\
f_{a}(y)+\varepsilon(x-y)
\end{array}\right\}
$$

of two symmetrically coupled logistic maps $f_{a}(x)=\operatorname{ax}(1$ $-x)$ with $3<a \leqslant 4$ and $-2 \leqslant \varepsilon \leqslant 2$. It is well known that the logistic map $f_{a}(x)$ for $a>a^{*} \cong 3.567 \ldots$ (the Feigenbaum accumulation point) undergoes a reverse cascade of homoclinic bifurcations of period- $2^{n}$ cycles at the parameter values $a_{n}$. At $a=a_{0} \cong 3.678573 \ldots$, for instance, the fixed 
point $x_{0}=1-1 / a$ undergoes its first homoclinic bifurcation, and two chaotic bands merge into a single band. Likewise, for $a=a_{1} \cong 3.592572 \ldots$, the period- 2 cycle undergoes its first homoclinic bifurcation, and four chaotic bands merge into two. At each of these homoclinic bifurcation points, $f_{a}(x)$ has a finite number of intervals with an absolutely continuous invariant measure. Hence the dynamics of $f_{a}(x)$ is chaotic. The main diagonal $\{x=y\}$ is a one-dimensional synchronization manifold for $F$.

In order to delineate the regions of parameter space in which the synchronized chaotic state is asymptotically stable, we have previously considered the transverse stability for each of the most important low-periodic point cycles $\gamma_{n}$ [16]. For $a=a_{0}$ we have found that the interval of asymptotic stability is bounded by a transverse perioddoubling bifurcation of the period- 6 cycle at $\varepsilon \cong-1.31$ and by a transverse period-doubling of the period-2 cycle at $\varepsilon$ $\cong-1.24$. For $a=a_{1}$, the interval of asymptotic stability for the synchronized chaotic state stretches from $\varepsilon \cong-1.46$ to $\varepsilon \cong-1.16$, with both the upper and the lower end being associated with a destabilization of the period- 2 cycle. The transverse destabilization of the fixed point is not associated with any riddling bifurcation in our model.

Let us examine the bifurcations in which the period-2 cycle

$$
x_{1,2}=y_{1,2}=\frac{a+1 \pm \sqrt{(a+1)(a-3)}}{2 a}
$$

loses its transverse stability. Results for cycles of arbitrary periodicity can be found in Ref. [21]. The map $F: \mathcal{R}^{2} \rightarrow \mathcal{R}^{2}$ is noninvertible, and it is easy to see that the Jacobian determinant $|D F|$ vanishes along two branches of a hyperbola (the so-called critical curves $[19,20])$

$$
L_{0}=\left\{(x, y): y=\frac{a-\varepsilon}{2 a}+\frac{\varepsilon^{2}}{4 a^{2}\left(x-\frac{a-2}{2 a}\right)}\right\}
$$

that intersect the diagonal $\{x=y\}$ in the points $x_{c 1}=1 / 2$ and $x_{c 2}=1 / 2-/ \varepsilon / a$. It follows that $F$ is a diffeomorphism in a neighborhood of the points $P_{i}(i=1$ and 2$)$ of the symmetric period-2 cycle, provided that $a \neq 1+\sqrt{5}$ and $a \neq 1$ $+\sqrt{4+(1+2 \varepsilon)^{2}}$. These provisions also imply that $\nu_{\|} \neq 0$ and $\nu_{\perp} \neq 0$, where

$$
\nu_{\|}=f^{\prime}\left(x_{1}\right) f^{\prime}\left(x_{2}\right)=1-(a+1)(a-3)
$$

and

$\nu_{\perp}=\left(f^{\prime}\left(x_{1}\right)-2 \varepsilon\right)\left(f^{\prime}\left(x_{2}\right)-2 \varepsilon\right)=(1+2 \varepsilon)^{2}-(a+1)(a-3)$

are the longitudinal and transverse eigenvalues for the period-2 cycle. $f^{\prime}(x)$ denotes the derivative of $f(x)$. Moreover, for $\varepsilon \neq 0$ and $\varepsilon \neq-1$, the first nonresonant condition $\nu_{\|} \neq \nu_{\perp}$ will be satisfied. For each of the two points $P_{i}$ on the period-2 cycle a neighborhood will then exist in which the transverse invariant manifolds $W_{\perp i}$ are at least $C^{1}$ smooth [23]. Invariance in this case obviously applies with respect to the iterated map $F^{2}$.
In the parameter interval of interest, the symmetric period- 2 cycle is unstable in the direction of the longitudinal manifold $W_{\|}=\{x=y\}$. The cycle loses its transverse stability either in a pitchfork bifurcation $\left(\nu_{\perp}=1\right)$ for

$$
\varepsilon=-\frac{1}{2}[1 \pm \sqrt{(a+1)(a-3)+1}]
$$

or in a period-doubling bifurcation $\left(\nu_{\perp}=-1\right)$ for

$$
\varepsilon=-\frac{1}{2}[1 \pm \sqrt{(a+1)(a-3)-1}] .
$$

To investigate how map (2.1) acts along the transverse manifolds $W_{\perp i}$ we rewrite $F$ in terms of the new variables $\xi=(x+y) / 2$ and $\eta=(y-x) / 2$

$$
\widetilde{F}:\left\{\begin{array}{c}
\xi \\
\eta
\end{array}\right\} \rightarrow\left\{\begin{array}{c}
f(\xi)-a \eta^{2} \\
\left(f^{\prime}(\xi)-2 \varepsilon\right) \eta
\end{array}\right\}
$$

where, as before, $f=f_{a}$. The term transverse manifold is meant to denote the manifold in which the asynchronous cycle(s) involved in the bifurcation is (are) situated. In the neighborhood of each period-2 cycle point $P_{i}$ we can expand the one-dimensional manifolds $W_{\perp i}=\left\{(\xi, \eta): \xi=\varphi_{i}(\eta)\right\}$ such that

$$
\varphi_{i}(\eta)=x_{i}+B_{i} \eta^{2}+(\text { higher order terms })
$$

Linear contributions to $\varphi_{i}$ vanish because $W_{\perp i}$ is parallel to the $\eta$-axis for $\eta=0$.

The coefficients $B_{i}$ may be obtained by inserting Eq. (2.9) into Eq. (2.8), and using the invariance of $W_{\perp i}$. This gives

$$
B_{i}=a\left[\nu_{\|, i+1}+\nu_{\perp, i}^{2}\right] /\left(\nu_{\|}-\nu_{\perp}^{2}\right),
$$

where $\nu_{\|, i}=f^{\prime}\left(x_{i}\right), \quad \nu_{\perp, i}=f^{\prime}\left(x_{i}\right)-2 \varepsilon$, and $x_{i+1}=f\left(x_{i}\right)$. From Eq. (2.10), $B_{i}$ can be calculated provided that the second nonresonant condition $\nu_{\|} \neq \mathrm{v}_{\perp}^{2}$ is satisfied. This condition, which guarantees the $C^{2}$ smoothness of $W_{\perp i}$, will always be fulfilled since $\nu_{\|}<0$ in the chaotic regime $a>a^{*}$.

The one-dimensional mapping $h_{i}: W_{\perp i} \rightarrow W_{\perp i}$ of $F^{2}$ along the transverse manifolds of the period-2 cycle takes the asymptotic form

$$
h_{i}: \eta \rightarrow \nu_{\perp} \eta+C_{i} \eta^{3}+(\text { higher order terms }) .
$$

Quadratic terms do not arise in this expansion because of the symmetry of the system. Inserting Eq. (2.11) into Eq. (2.8) and using our results (2.10) for $B_{i}$ we obtain

$$
C_{i}=-2 a^{2}\left[\frac{\left(\nu_{\perp, i}^{2}+\nu_{\|, i+1}\right)\left(\nu_{\perp, i+1}+\nu_{\|, i} \nu_{\perp, i}\right)}{\nu_{\|}-\nu_{\perp}^{2}}-\nu_{\perp, i}\right] .
$$

It is well known from normal form theory that the bifurcations of the symmetric one-dimensional map $h_{i}$ will be supercritical or subcritical depending on the sign of the product $\nu_{\perp} C_{i}$. (Obviously, $C_{i}$ must have the same sign for the two transverse manifolds.) If $\nu_{\perp} C_{i}<0$, the bifurcation is supercritical, and it is subcritical for $\nu_{\perp} C_{i}>0$. Direct calculation shows that $C_{i}$ is positive in the relevant parameter interval. Hence we conclude that the transverse pitchfork bifurcation of the symmetric period-2 cycle occurring at $\varepsilon$ 


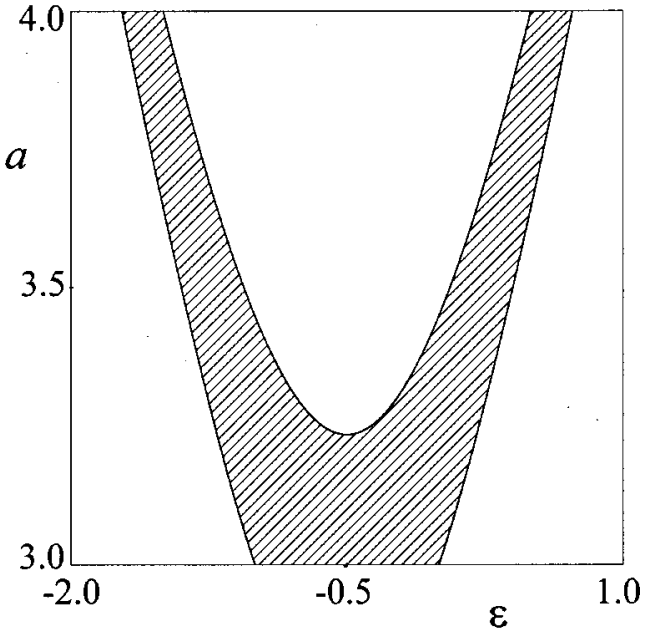

FIG. 1. Region of transverse stability for the symmetric period-2 cycle. Destabilization occurs via a subcritical pitchfork bifurcation (lower curve) or via a supercritical period-doubling bifurcation (upper curve). Stability regions for other low-periodic cycles may be found in Ref. [16]. For our discussion of chaotic synchronization, only the region $a>a^{*} \cong 3.569$ is of interest.

$=-\frac{1}{2}[1 \pm \sqrt{(a+1)(a-3)+1}]$ is subcritical, and the transverse period-doubling occurring at $\varepsilon=-\frac{1}{2}[1$ $\pm \sqrt{(a+1)(a-3)-1}]$ is supercritical. As an illustration to this discussion, Fig. 1 shows the region in parameter space in which the symmetric period-2 cycle is transversely stable. This region is delineated by the curves (2.6) and (2.7) along which the subcritical pitchfork and the supercritical perioddoubling bifurcations take place. In the interval $3<a<1$ $+\sqrt{6}$, synchronization of the period- 2 cycle occurs for arbitrary small values of the coupling parameter. This is the interval in which the period-2 cycle $x_{2}=f\left(x_{1}\right), x_{1}=f\left(x_{2}\right)$ is the only existing solution for the individual map. For $\varepsilon=0$, the two-map system displays a synchronous period- 2 cycle $\left(x_{1}, x_{1}\right) \rightarrow\left(x_{2}, x_{2}\right) \rightarrow\left(x_{1}, x_{1}\right)$ and an antisymmetric period-2 cycle $\left(x_{1}, x_{2}\right) \rightarrow\left(x_{2}, x_{1}\right) \rightarrow\left(x_{1}, x_{2}\right)$. Both of these cycles are stable, and their basins of attraction are organized in a characteristic chessboard structure. Moreover, except near the ends of the stability interval $3<a<1+\sqrt{6}$, both cycles remain stable as $\varepsilon$ attains a small (positive or negative) value.

The bifurcation diagram in Fig. 2(a) unfolds the supercritical transverse period-doubling bifurcations of the synchronous period-2 cycle. Here, $a=a_{0}$. To the left and right in the diagram the fully drawn horizontal lines denote the transversely stable period-2 cycle. In each of the perioddoubling bifurcations, the period-2 saddle (denoted $P_{2}$ ) is turned into a repeller, and a symmetric period- 4 saddle cycle (denoted $P_{4}$ ) is born with its unstable manifolds along the main diagonal $\{x=y\}$. The figure shows how the same period-4 cycle is involved in both bifurcations. This is even more clear from the phase portrait in Fig. 2(b) where we can follow how the period-4 saddle as the coupling parameter is increased moves out in phase space to return to the synchronous period-2 cycle. On the way, as shown in Fig. 2(a), the period-4 cycle undergoes a couple of saddle-node bifurcations. As we shall see below, the period-4 cycle is also involved in a number of additional bifurcations through which it may stabilize in certain regions of parameter space.
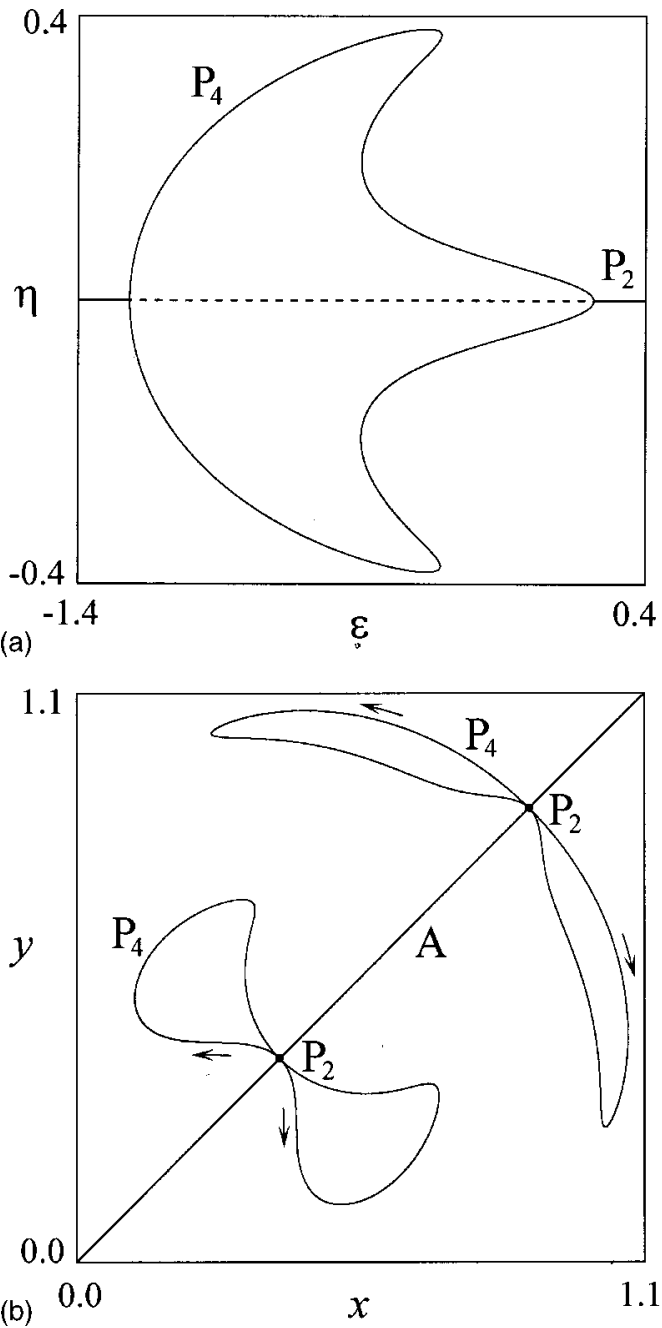

FIG. 2. (a) Bifurcation diagram for the supercritical transverse period-doubling bifurcations of the symmetric period-2 cycle. (b) Corresponding phase portrait showing how the position of the period-4 saddle $\left(P_{4}\right)$ varies as the coupling parameter is increased. The figure was obtained for $a=a_{0}$.

Figures 3(a) and 3(b) show a similar set of diagrams for the subcritical transverse pitchfork bifurcation of the synchronous period-2 cycle that occurs at $\varepsilon=-\frac{1}{2}[1$ $+\sqrt{(a+1)(a-3)+1}]$. The two fully drawn line segments along the horizontal axis of Fig. 3(a) again represent the regions of transverse stability for the period- 2 cycle. The pitchfork bifurcation takes place at the left hand edge of the left of these intervals. The curves denoted $P_{1}$ follow two symmetric asynchronous period-1 orbits produced in a transverse pitchfork bifurcation of the synchronous fixed point $P\left(x_{0}, x_{0}\right), x_{0}=1-1 / a$ at $\varepsilon=-(a-1) / 2$ and connecting to the fixed point at $(0,0)$. The figures show how two mutually symmetric period-2 repellers (denoted $P_{2}$ ) approach the synchronous period- 2 saddle from either side to annihilate with one another and transform the saddle into a repeller. To the right hand side of the bifurcation diagram the period- 2 repellers undergo an inverse period-doubling (marked PD) producing the above mentioned symmetric period-1 repellers.

Immediately after its birth, the points of the period-4 cycle in Fig. 2(a) move away from the diagonal in accordance with the usual square root relation 

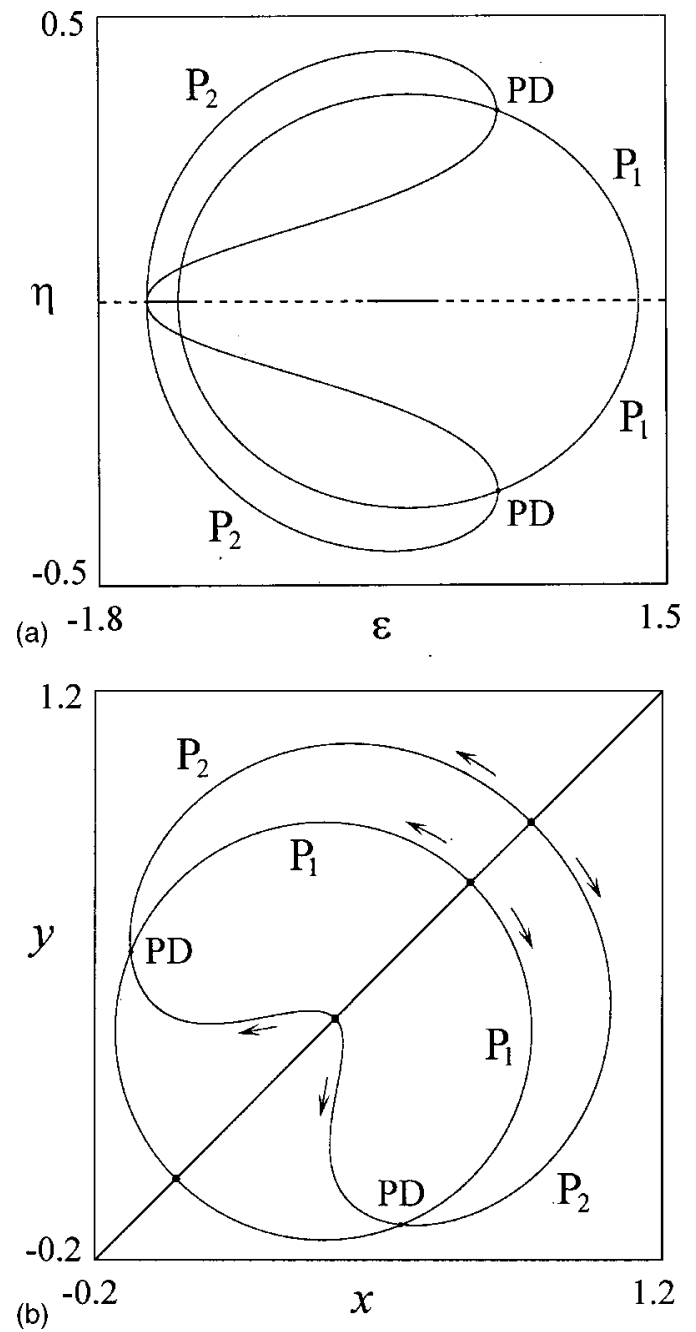

FIG. 3. (a) Bifurcation diagram for one of the subcritical transverse pitchfork bifurcations of the symmetric period-2 cycle. (b) Corresponding phase portrait. The curves marked $P_{1}$ and $P_{2}$, respectively, show how pairs of mutually symmetric period- 1 and -2 repellers move under variation of the coupling parameter.

$$
|\eta| \sim\left|\varepsilon-\varepsilon_{c}\right|^{1 / 2}
$$

where $\varepsilon_{c}$ denotes the bifurcation point and $\left|\varepsilon-\varepsilon_{c}\right| \ll 1$. This result hinges on the fact that $\varepsilon$ is a normal parameter, i.e., that changes in $\varepsilon$ do not affect the dynamics of the synchronous state. It is worth noticing that although the parameters $C_{i}, i=1$, and 2 , have the same sign at the point of bifurcation, in general they do not have the same magnitude. As we can see [Fig. 2(b)], this implies that the two point pairs of the asynchronous period- 4 cycle do not move out along their respective manifolds at the same speed.

Figure 4 shows the results of a numerical evaluation of the coefficients $C_{1}$ for the upper point of the period- 2 cycle. The two curves denoted $C_{1}^{\mathrm{PF}}$ and $C_{1}^{\mathrm{PD}}$ refer to the transverse destabilization via a pitchfork and a period-doubling bifurcation, respectively. (It is a simple matter to obtain analytic expressions for these curves. However, the expressions are a little clumsy, and we have left them out here). The curve for $C_{1}^{\mathrm{PF}}$ passes through zero for $a=3$ where the symmetric period-2 cycle first arises. The curve for $C_{1}^{\mathrm{PD}}$ diverges for $a=1+\sqrt{5} \cong 3.236$, where the longitudinal eigenvalue $\nu_{\|}$

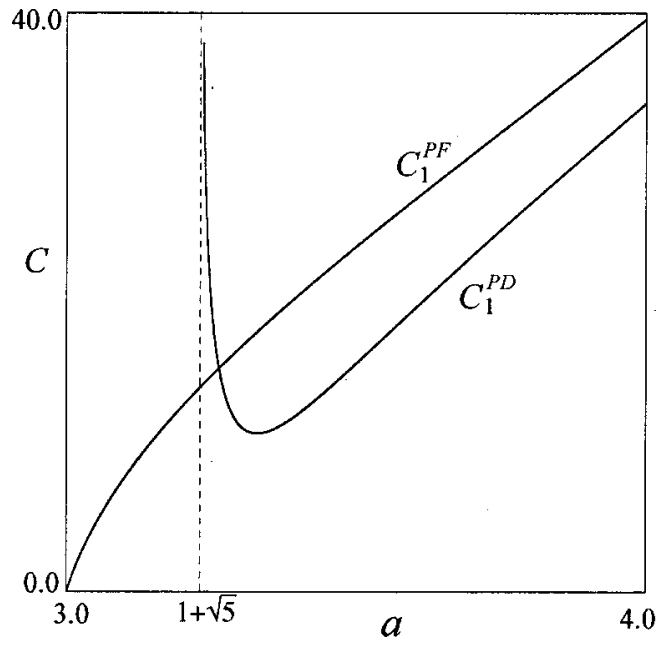

FIG. 4. Variation of the coefficients $C_{1}^{\mathrm{PF}}$ and $C_{1}^{\mathrm{PD}}$ associated, respectively, with the transverse pitchfork and the transverse period-doubling bifurcations of the symmetric period-2 cycle.

vanishes. This is the point where the individual map $f_{a}(x)$ displays a superstable period-2 orbit. It is also the minimal value of $a$ for which a transverse period-doubling bifurcation can occur (see Fig. 1).

Remark: The above analysis has assumed the interacting maps to be identical. In the presence of a small parameter mismatch a fully symmetric period-2 cycle can no longer exist, and the pitchfork bifurcation will be replaced by a saddle-node bifurcation, involving the simultaneous appearance or disappearance of a repeller and a saddle cycle slightly off the main diagonal. For the period-doubling bifurcation the condition for a subcritical or supercritical transition will be determined by the sign of the Schwarzian derivative $S h=h^{\prime \prime \prime} / h^{\prime}-(3 / 2)\left(h^{\prime \prime} / h^{\prime}\right)^{2}$ of the map $h$ along the transversal manifold. If, in the moment of bifurcation $S h$ $<0$, then the bifurcation is supercritical. If $S h>0$, it is subcritical. The simplification we have used for the symmetric case is to take $h^{\prime \prime}=0$.

\section{EXAMPLE OF A SOFT RIDDLING BIFURCATION}

Figure 5 illustrates the situation in the phase plane of our coupled map system for $a=a_{0}$ and $\varepsilon=-1.234$. With this value of $a$ the transverse period doubling of the period-2 cycle occurs at $\varepsilon \cong-1.2373$. The full line along the diagonal represents the synchronized chaotic state, and the two points on this line indicated by open circles are the points of the period-2 cycle that has just undergone a transverse period doubling. In the vicinity of these points, the four points indicated by circles with crosses through are the points of the asynchronous period- 4 cycle that has appeared in the bifurcation. The period- 4 cycle is a saddle cycle with a stable manifold that connects it with the period-2 repeller. The unstable manifolds of the period- 4 saddle stretch along the synchronization manifold. The arrows on the various stable and unstable manifolds denote their forward directions. It should be noted that since we are dealing with a noninvertible map, a (stable or unstable) manifold is allowed to intersect itself. Note how the two point pairs of the period-4 cycle, in accordance with the above discussion, have moved different distances away from the period- 2 cycle. Also shown in Fig. 5 is 


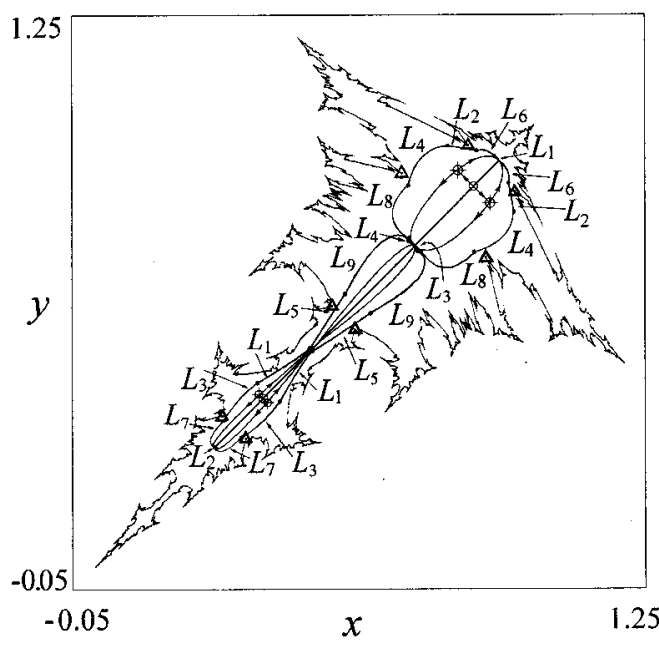

FIG. 5. Situation in the phase plane immediately after the supercritical transverse period-doubling of the symmetric period-2 cycle. The figure shows the period- 4 saddle cycle with its stable and unstable manifolds. Also indicated are the absorbing area as bounded by segments of critical curves $L_{k}$ and the basin of attraction with its fractal boundary. $\varepsilon=-1.234$.

the fractal boundary of the basin of attraction for the synchronized chaotic state.

In order to demonstrate a number of important details, Fig. 6 shows a magnification of the upper part of the phase plane. Here $\varepsilon=-1.225$. $A$ denotes (part of) the synchronized chaotic set, and $P_{2}$ and $P_{4}$ are points on the period-2 repeller and the period- 4 saddle cycle, respectively.

As bounded by the outmost loops of the unstable manifolds for the period- 4 cycle (drawn as thin lines) and by segments of the critical curves $L_{1}-L_{8}$ (drawn as heavier

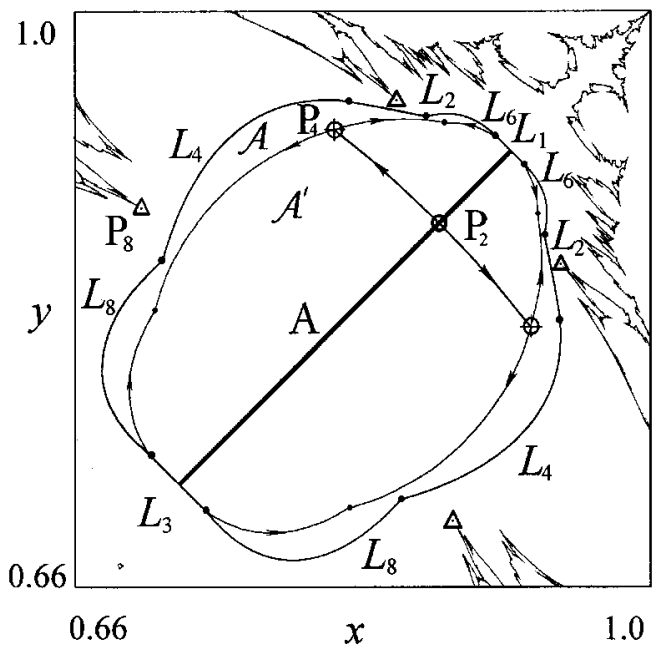

FIG. 6. Magnification of the upper part of Fig. 5. Here the coupling parameter is $\varepsilon=-1.225$. A denotes (part of) the synchronized chaotic set, and $P_{2}$ and $P_{4}$ are points of the period-2 repeller and the period- 4 saddle cycle, respectively. Points $P_{8}$ denoted by a small triangle belong to a period- 8 repeller, which is situated at the boundary of the basin of attraction. lines), the mixed absorbing area $\mathcal{A}^{\prime}[19,24]$ completely surrounds the synchronized chaotic set. With the situation depicted in Fig. 6, the mixed absorbing area is a closed invariant set, i.e., $F\left(\mathcal{A}^{\prime}\right)=\mathcal{A}^{\prime}$. Hence trajectories starting inside the mixed absorbing area (or at its boundary) cannot leave it. Moreover, most trajectories starting from a neighborhood outside $\mathcal{A}^{\prime}$ will follow the unstable manifolds of the period-4 saddle, fold at the critical curves $\left(L_{1}\right.$ or $\left.L_{3}\right)$, and cross into $\mathcal{A}^{\prime}$. We conclude that as long as a mixed absorbing area exists inside the basin of attraction (and no other attractor exists within the mixed absorbing area), the synchronized chaotic state will attract almost all points from its neighborhood. $\mathcal{A}$ is then a Milnor attractor, and its basin of attraction can be locally riddled only [16]. In this way the formation in a supercritical transverse bifurcation of a mixed absorbing area that stretches along the synchronized chaotic state plays a major role for restraining the amplitude of the burst away from the synchronized state.

Surrounding the mixed absorbing area in Fig. 6, we find the absorbing area $\mathcal{A}$ (also referred to as the trapping region [10]). Bounded by a finite number of segments $L_{k}=F^{k}\left(L_{0}\right)$ of images of the critical curves $L_{0}$ as given by Eq. (2.3), the absorbing area $[19,20]$ has the property that trajectories that enter this area cannot leave it again, i.e., $F(\mathcal{A}) \subseteq \mathcal{A}$. Moreover, trajectories that start in the neighborhood of the absorbing area will enter it in a finite number of iterations. The presence of an absorbing area is a characteristic feature of noninvertible maps. For our system of two coupled logistic maps, the absorbing area exists in an interval of the coupling parameter that includes part of the interval of asymptotic stability for the synchronized chaotic state, and can stretch beyond this interval. When, as illustrated in Fig. 6, the mixed absorbing area falls fully within the absorbing area (and no other attracting states than $A$ exists in $\mathcal{A}$, almost all trajectories starting in the absorbing area will end up in the mixed absorbing area. Finally, on the boundary of the basin of attraction (and indicated by small triangles in Fig. 6) we find the points $P_{8}$ of a period-8 repeller. Any neighborhood of this repeller contains a positive measure set of initial conditions from which the trajectories diverge towards infinity.

As we continue to increase the coupling parameter, the points $P_{4}$ of the period-4 saddle cycle move further out along the transverse manifolds of the period-2 repeller, and the mixed absorbing area $\mathcal{A}^{\prime}$ continues to grow until it fills out most of the absorbing area $\mathcal{A}$. This is illustrated in Fig. 7, where $\varepsilon=-1.21$. The critical curves $L_{k}$ will serve as an envelope to the unstable manifolds, and as long as the period-4 cycle falls within the boundary of the absorbing area, its unstable manifolds will be restrained to this area. Compared with Fig. 6, the period-8 repeller (and hence the basin boundary) has moved closer to the boundary of the absorbing area. At $\varepsilon \cong-1.205$ a crisis takes place as the boundary of the absorbing area comes in contact with the basin boundary, and the period- 8 repeller crosses into the region delineated by the critical curves. We can see that this happens in points where the boundaries of $\mathcal{A}$ and $\mathcal{A}^{\prime}$ coincide. This marks the transition from local to global riddling of the basin of attraction [16].

Figure 8 shows the situation immediately after this contact bifurcation has occurred. Here $\varepsilon=-1.18$. The points of the period- 8 repeller now fall inside the region delineated by 


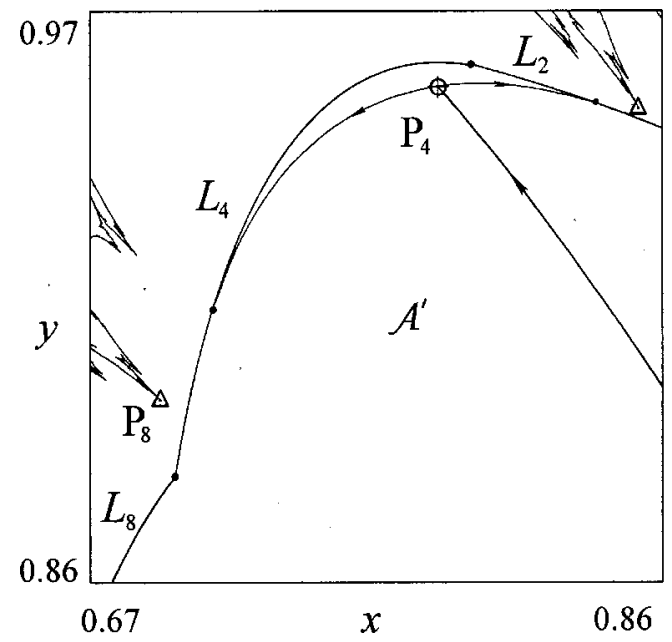

FIG. 7. For $\varepsilon=-1.21$, the period-4 saddle cycle has moved further out along the transverse manifolds of the period-2 repeller, and the mixed absorbing area $\mathcal{A}^{\prime}$ covers most of the absorbing area $\mathcal{A}$. At the same time the period- 8 repeller has moved closer to the common boundary of $\mathcal{A}$ and $\mathcal{A}^{\prime}$.

the images of the critical curves, the absorbing area has ceased to exist, and direct access has been opened for points starting near the transversely unstable period- 2 cycle (and its dense set of preimages) to diverge to infinity. This is a typical example of global riddling. One can examine this transition in more detail by constructing the preimages of those tongues of the basin of infinity that have penetrated into the region $\mathcal{A} \cap \mathcal{A}^{\prime}$. Immediately after the boundary crisis these preimages can be followed backward along the unstable manifolds that define the boundary of $\mathcal{A}^{\prime}$ toward the period-4 saddle cycle, and from here along the transverse manifolds back towards the period- 2 repeller on the main diagonal.

Remark: To complete the present discussion, let us note that while varying the coupling parameter from the point of

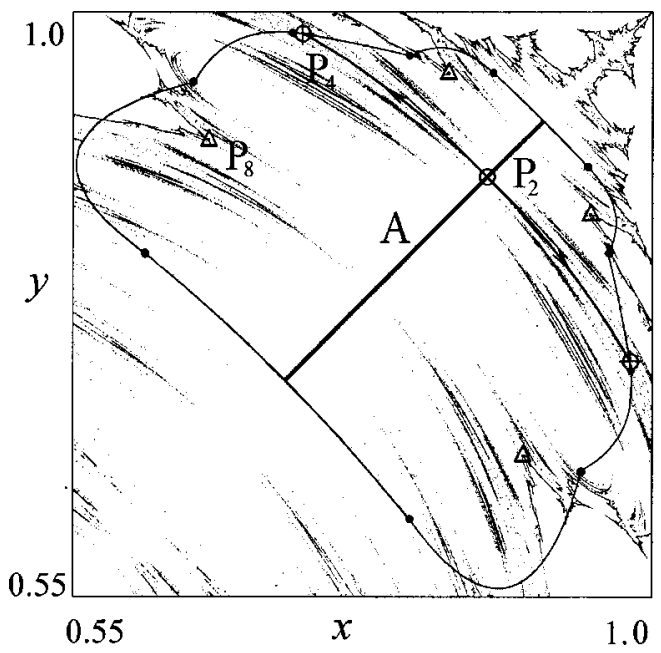

FIG. 8. Crossing of the period-8 repeller through the segments of critical curves and unstable manifolds that define $\mathcal{A}$, and $\mathcal{A}^{\prime}$ marks the transition from locally to globally riddled basins of attraction. There is now direct access from the neighborhood of the transversely unstable period- 2 cycle (and its dense set of preimages) to infinity. transverse destabilization of the period-2 cycle to the final boundary crisis, we have not found evidence of the presence of other attracting sets in $\mathcal{A}^{\prime}$ besides the synchronized chaotic state. This is the reason why we have been able to say that the basin of attraction for $\mathcal{A}$ is locally riddled. However, we cannot exclude that this is a result only of our finite numerical accuracy. Our simulations have revealed how the stable and unstable manifolds of the period-4 saddle intersect in many points. This implies the existence of homoclinic trajectories and of the complicated dynamics associated with a Smale horseshoe. It is well known that under specific conditions this can lead to the formation of Newhouse regions in parameter space with a dense set of systems having infinitely many attracting cycles $[25,26]$. The presence of such regions causes irreducible problems mathematically as well as numerically, and we shall not discuss their consequences here.

We consider the above scenario to describe the generic transition from locally to globally riddled basins of attraction following a supercritical riddling bifurcation in coupled map systems when the asynchronous cycle born in the bifurcation does not stabilize before the contact bifurcation between the absorbing area and the basin of attraction has occurred. Our analyses of this transition were recently criticized by Bischi and Gardini [22]. Unfortunately, these authors did not notice that when the contact bifurcation occurs, the boundaries of the absorbing and the mixed absorbing areas coincide. Hence the bifurcation involves a minimal, invariant absorbing area, and the criticism is not justified.

In Sec. $\mathrm{V}$ we shall discuss a very different and much more complicated scenario that arises for $a=a_{1} \cong 3.592572 \ldots$. This is the parameter value for which the period- 2 cycle of the individual map $f_{a}(x)$ displays its first homoclinic bifurcation. Here the asynchronous saddle cycle born in the riddling bifurcation stabilizes in an inverse period-doubling bifurcation to produce an attracting state inside the absorbing area for the synchronized chaotic set. First, however, let us consider a generic scenario for the appearance of globally riddled basins of attraction following a hard riddling bifurcation that can also be observed for $a=a_{0}$.

\section{SCENARIO FOR A HARD RIDDLING BIFURCATION}

At the other end of the interval of asymptotic stability for $a=a_{0}$, a transverse destabilization of the symmetric period-6 cycle takes place [16]. This again occurs via a period-doubling bifurcation $\left(\nu_{\perp}=-1\right)$. However, evaluation of the parameters $C_{i}$ using the procedure described in Ref. [21] shows that the bifurcation is subcritical $\left(C_{i}<0\right)$. Hence, before the transition occurs, the points of a period-12 repeller are situated on either side of the symmetric period-6 cycle. This situation is illustrated in Fig. 9(a) where we also can see the fractal boundary of the basin of attraction for the synchronized chaotic state. Closer examination reveals that the points of the period- 12 repeller fall at cusp points of the basin boundary, and any neighborhood of these points contains a positive measure set of initial conditions from which the trajectories diverge to infinity.

Figure $9(\mathrm{~b})$ is a magnification of part of the structure in Fig. 9(a). Here the coupling parameter $\varepsilon=-1.3$. The figure shows (the upper part of) the synchronous chaotic state with three points of the symmetric period- 6 cycle situated along 

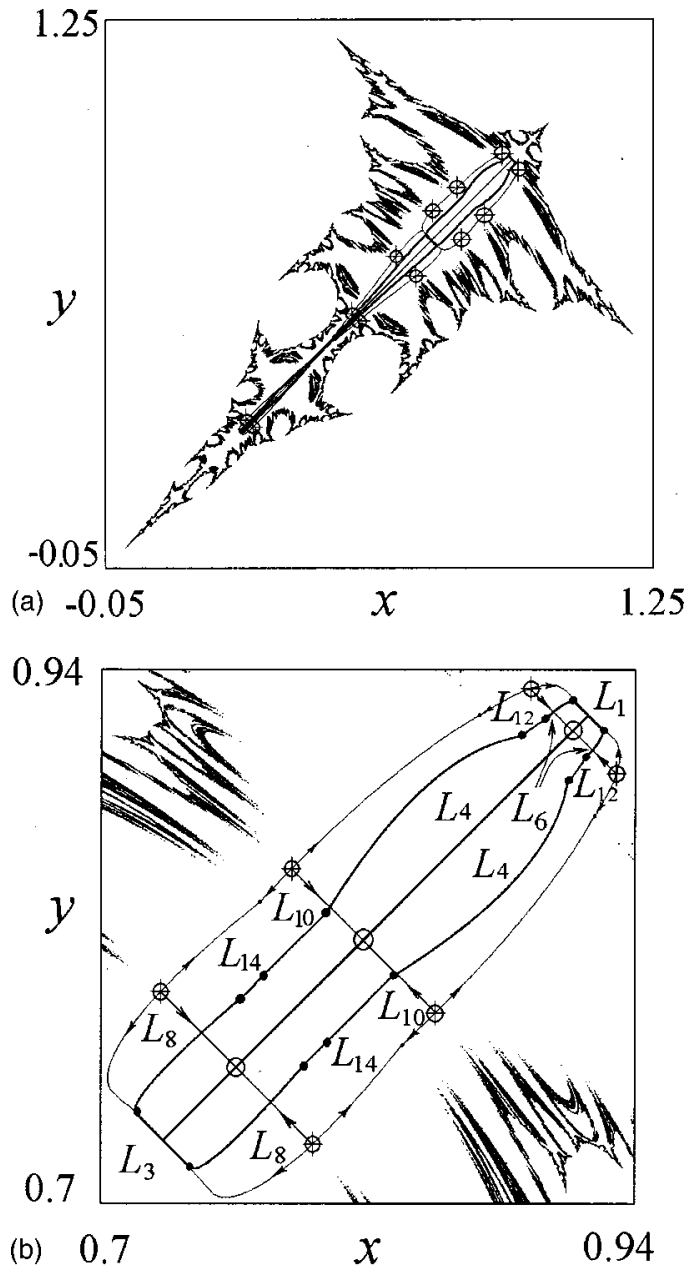

FIG. 9. (a) Overview of the situation in phase plane before the transverse destabilization of the symmetric period- 6 cycle. The figure shows the asymmetric period-12 repeller with its longitudinal manifolds. (b) Magnification of part of (a). Note how the absorbing area falls inside the mixed absorbing area, which now exists before the riddling bifurcation.

the main diagonal. Also shown is the absorbing area as bounded by segments of the critical curves $L_{1}-L_{14}$ (heavy lines). The dots indicate where these segments connect. Outside the absorbing area we find six points of the period-12 repeller (shown as circles with crosses through). As noted above, these points fall at cusp points of the basin boundary. In the longitudinal direction the unstable manifolds of the period-12 repeller again seem to wind around the synchronized chaotic state. However, since these manifolds now fall outside the absorbing area, we cannot be sure that they will always remain bounded in the vicinity of the synchronous set. In the present situation this appears to be the case, since the unstable longitudinal manifolds all connect to the critical curves and hence define a mixed absorbing area (which now exists before the riddling bifurcation).

As we approach the bifurcation, the points of the period-12 repeller move through the critical curves $L_{k}$, and the absorbing area ceases to exist. Unstable manifolds now point out through the images of the critical curves, and trajectories starting outside of the longitudinal manifolds of the period-12 repeller will diverge. This situation is illustrated in Fig. 10 for $\varepsilon=-1.307$. Finally, at the point of bifurcation

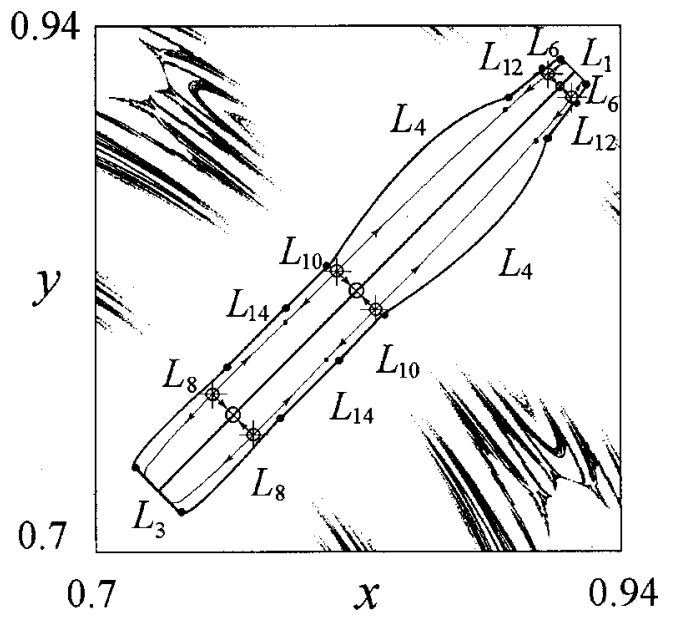

FIG. 10. For $\varepsilon=-1.307$ the period-12 repeller has moved across the critical curves, and the absorbing area has ceased to exist.

where the period-12 repeller disappears, direct access is opened from the symmetric period-6 cycle (as well as from its dense set of preimages) to infinity. In accordance with the scenario described by Lai et al. [15], the emergence of global riddling in this way occurs simultaneously with the destabilization of the period- 6 cycle in a local bifurcation.

Based on the above results we conclude that the riddling bifurcation will be hard if it is associated with a subcritical destabilization of some orbit. The subcritical bifurcation does not produce asynchronous saddle cycles whose unstable manifolds can restrain the bursts of trajectories. It is possible, of course, that saddle cycle(s) of appropriate periodicity could exist outside the repelling cycle(s) involved in the transverse destabilization (an example was provided by the model considered in Ref [18]). In this case the subcritical riddling bifurcation might not directly produce a globally riddled basin of attraction.

\section{DESTABILIZATION SCENARIO FOR $a=a_{1}$}

Our last example concerns the sequence of events that take place in connection with the destabilization of the synchronized chaotic state for $a=a_{1}$ and $\varepsilon \cong-1.1$. This scenario starts with the transverse destabilization of the symmetric period-2 cycle in a supercritical period-doubling bifurcation for $\varepsilon \cong-1.1560$. The blowout bifurcation at which the transverse Lyapunov exponent becomes positive, and the chaotic state loses its average attraction occurs at $\varepsilon$ $\cong-1.0385$. Hence we are interested in following the bifurcations that take place in the interval between $\varepsilon \cong-1.1560$ and -1.0385 .

For $a=a_{1}$, the synchronous chaotic state consists of two separate bands. The absorbing area and mixed absorbing area each therefore also consists of two regions that are mapped one into the other under the action of $F$. Figure 11(a) shows the upper part of the phase plane after the transverse perioddoubling has occurred. Here $\varepsilon=-1.155$. We note the two points of the asynchronous period- 4 saddle cycle situated along the transverse manifolds of the symmetric period-2 repeller. The points of the period- 4 saddle are indicated as circles with crosses through. Together with segments of the critical curves $L_{1}$ and $L_{3}$, the unstable manifolds of the 

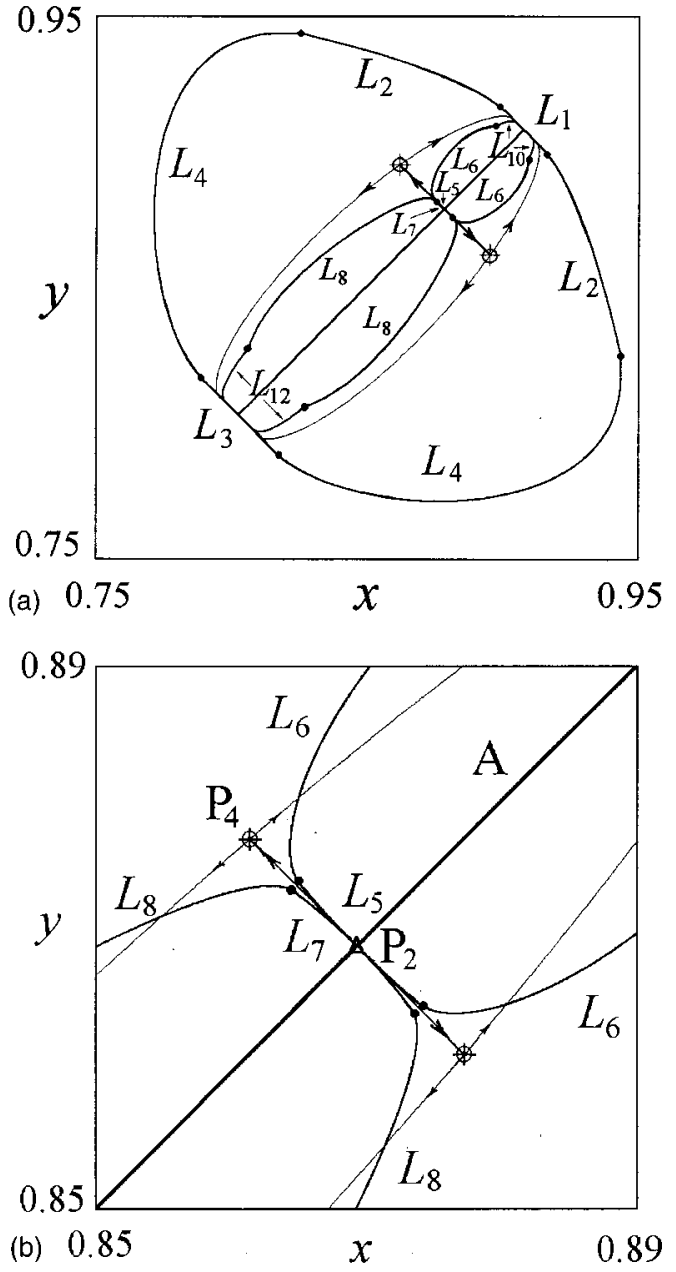

FIG. 11. (a) Upper part of the phase plane for $a=a_{1}$ and $\varepsilon=$ -1.155 . We observe a larger and a smaller absorbing area together with a mixed absorbing area delineated by the unstable manifolds of the asymmetric period-4 saddle. (b) Magnification of part of the phase plane for $\varepsilon=-1.15597$. Note how the transverse manifolds of the period-2 repeller pass right between the critical curves.

period-4 saddle define a mixed absorbing area $\mathcal{A}^{\prime}$. Around this area we find a large absorbing area $\mathcal{A}$ as bounded by segments of the iterates $L_{1}, L_{2}, L_{3}$, and $L_{4}$ of $L_{0}$.

Trajectories starting inside the mixed absorbing area cannot escape from it. Except for a measure zero set of trajectories starting from points on the external branches of the stable manifolds of the asynchronous period- 4 cycle (and possible preimages of these manifolds), trajectories starting from a neighborhood of the mixed absorbing area will move along the unstable manifolds of the period- 4 saddle points toward the points where they meet the critical curve $L_{3}$ (or $L_{1}$ ). After a folding here they will be mapped into the mixed absorbing area to soon be trapped in the smaller absorbing area defined by the critical curves $L_{1}, L_{3}, L_{5}, L_{7}, L_{6}, L_{8}$, $L_{10}$, and $L_{12}$. The transverse manifolds of the synchronous period-2 cycle pass right between the critical curves $L_{5}$ and $L_{7}$. Hence, in spite of the fact that the period- 2 cycle has a dense set of preimages along the main diagonal, not a single trajectory will be able to leave the smaller absorbing area.

Remark: The smaller absorbing area represents the absorbing area that existed before the homoclinic bifurcation in which the four-band chaotic attractor for the individual map

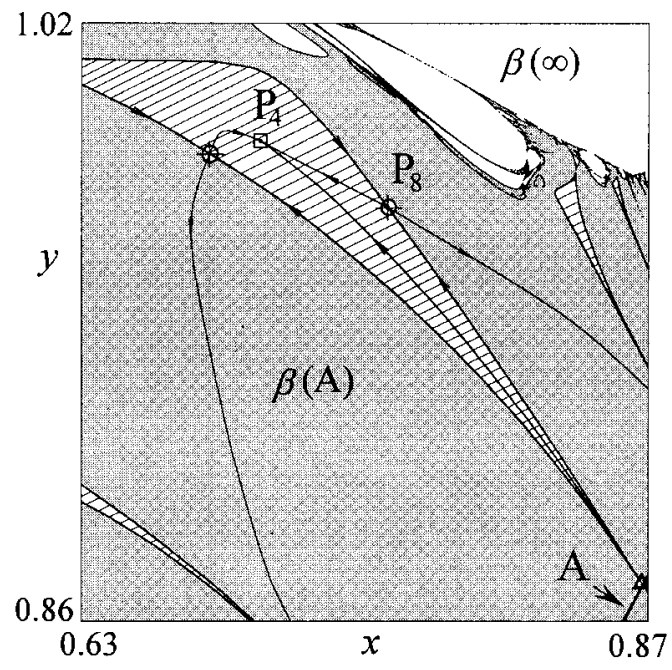

FIG. 12. The asymmetric period-4 cycle has undergone an inverse, subcritical period-doubling transition, producing a stable period-4 cycle $P_{4}$ and a period- 8 saddle $P_{8}$. The stable manifolds of this saddle delineate the immediate basin for the period-4 node. The figure was obtained for $\varepsilon=-1.08$.

has merged into a two-band attractor. The smaller absorbing area is destroyed in the homoclinic bifurcation, and as soon as the nonlinearity parameter $a$ exceeds $a_{1}$ by as little as a part in $10^{11}$ one can start to observe how trajectories escape along the transverse manifold of the synchronous period-2 cycle.

Immediately after the transverse destabilization of the period-2 cycle [e.g., for $\varepsilon=-1.15597$ as shown in Fig. 11(b)] the period-4 saddle points will be situated very close to the main diagonal and its unstable manifolds stretch along the synchronized chaotic state as a narrow band from which trajectories cannot escape. Hence we again observe that a supercritical transverse bifurcation leads to a soft riddling transition with a locally riddled basin of attraction and with small and smoothly growing bursts of trajectories away from the synchronized state. As we move further away from the bifurcation point, however, a completely different sequence of events takes place. At $\varepsilon \cong-1.09571$ the period-4 saddle cycle undergoes an inverse, subcritical period doubling in the direction of its unstable manifold. This produces a stable asynchronous period- 4 cycle surrounded on both sides by the points of a period- 8 saddle cycle.

This situation is illustrated in Fig. 12, where a point $P_{4}$ of the stable period- 4 cycle is marked by a small square and the neighboring points $P_{8}$ of the period- 8 saddle by circles with small crosses. Situated along the main diagonal and embedded in the synchronized chaotic attractor $A$, the upper point of the period-2 repeller has been indicated by a small triangle. Also shown in this figure are the stable and unstable manifolds of the period- 8 saddle. The white region $\beta(\infty)$ in the top right corner belongs to the basin of infinity. With a gray shading, the area $\beta(A)$ is the basin of attraction for the synchronized chaotic state. As in the previous figures, the boundary between these two basins is fractal.

The inverse period-doubling bifurcation at $\varepsilon \cong-1.09571$ has produced a new attracting state inside the former basin of attraction for the synchronized chaotic state. The immediate 
basin for the period-4 cycle (shown crosshatched in Fig. 12) is defined by the stable manifolds of the period- 8 saddle. Emanating from the symmetric period-2 repeller, these manifolds delineate a basin that stretches as a set of tongues all the way down to the main diagonal. Immediately after the stabilization of the period- 4 cycle these tongues will be very narrow and they will not intersect the smaller absorbing area. As a consequence trajectories that start within this area will not be able to reach the stable period- 4 cycle, and the basin of attraction for the synchronized chaotic state will remain locally riddled only.

Besides the immediate basin, the basin of attraction for the asynchronous period- 4 cycle also consists of a set of secondary tongues. Most prominent among these are the tongues that emanate from the points where the critical curves $L_{1}$ and $L_{3}$ intersect the main diagonal. These secondary tongues are also clearly visible (crosshatched) in Fig. 12. Like the primary tongues they are delineated by sharp and well-defined edges.

If the value of the nonlinearity parameter $a$ exceeds $a_{1}$ by the smallest amount, the smaller absorbing area ceases to exist. The basin of attraction for the asynchronous period-4 cycle will then include tongues that emanate from the dense set of preimages of the period-2 repeller in the synchronized chaotic set $A$, and the basin of attraction for $A$ will be globally riddled. In this case the transition to global riddling is accomplished via two local bifurcations: first the transverse supercritical period-doubling of the symmetric period-2 cycle and thereafter the stabilization of the asynchronous period-4 cycle in an inverse subcritical period-doubling. However, the new tongues tend to be extremely narrow, and in the numerical calculations they show up only as randomly scattered points within the area that has otherwise been assigned to $\beta(A)$.

A similar transition to global riddling has recently been described by Astakhov et al. [27] for a pair of nonlinearly coupled logistic maps. In their main scenario it is the fixed point that first undergoes a transverse destabilization in the form of a supercritical period doubling. After similar perioddoubling bifurcations of the symmetric period- $2,-4,-8$, and -16 cycles, the asynchronous period- 2 saddle cycle produced in the first transverse bifurcation stabilizes in an inverse, subcritical pitchfork bifurcation, giving way to a globally riddled basin of attraction for the synchronous chaotic state.

We would like to emphasize, however, that the globally riddled basins of attraction created through these processes have a fairly unusual structure. First, the main tongues are delineated by the stable manifolds of a saddle cycle (or a pair of saddle cycles). By contrast, in the commonly described form of global riddling $[10,11,16]$, the repelling tongues are defined only in terms of bundles of trajectories that follow an unstable manifold away from the synchronized chaotic state. In addition there is a prominent set of secondary tongues that also have sharp and well-defined edges. Finally, the remaining tongues form an extremely thin structure that shows up in the numerical calculations only as randomly scattered points from which the trajectories eventually reach the asymmetric point attractor.

As the coupling parameter is further increased, the phase portrait starts to become complicated. At $\varepsilon=-1.085$, the asynchronous period- 4 cycle is transformed from a stable

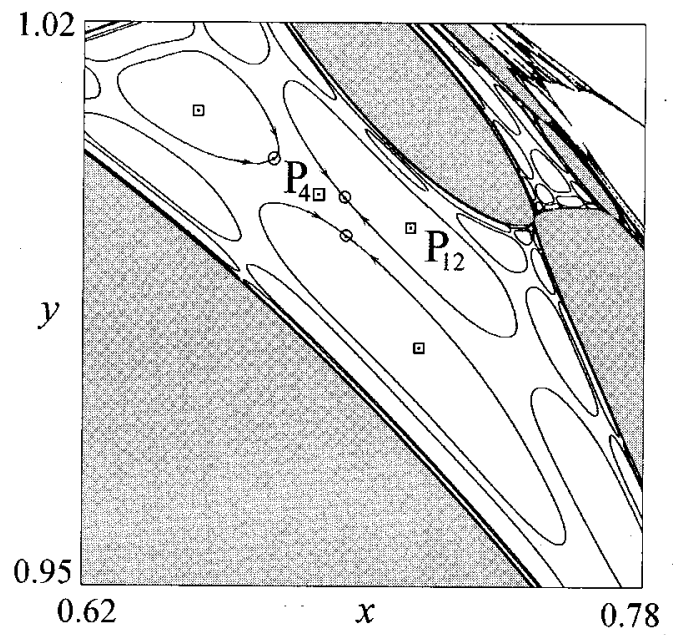

FIG. 13. A stable period-12 cycle has been born together with a period-12 saddle in a subharmonic saddle-node bifurcation. The stable manifold of the period-12 saddle defines discs shaped immediate basins for the period-12 node. Here $\varepsilon=-1.0615$.

node into a stable focus (i.e., the eigenvalues become complex conjugated), and at $\varepsilon \cong-1.0758$ the period- 8 saddle undergoes a supercritical pitchfork bifurcation in the direction of its stable manifold. This produces a period- 8 repeller surrounded by two period- 8 saddles at the boundary of the immediate basin of attraction for the period- 4 focus. At $\varepsilon$ $\cong-1.0625$ a subharmonic saddle-node bifurcation [28] takes place in which a stable period- 12 cycle is born together with a period-12 saddle. This situation is illustrated in Fig. 13 , where $\varepsilon=-1.0615$.

We now have two coexisting stable solutions in the repelling tongues emanating from the symmetric period-2 repeller. In Fig. 13, a point $P_{4}$ of the period-4 focus is indicated by a square drawn with relatively heavy lines, and the points $P_{12}$ of the period- 12 node are indicated by finer squares. The immediate basin of attraction for the period- 12 cycle takes a very unusual form, namely, as a set of discs surrounded by topological circles which are formed by the stable manifold of the period-12 saddle (points of which are shown as small circles). This peculiar structure, in which the same stable manifold as a closed curve approaches the saddle point from both sides, is made possible by virtue of the noninvertibility of the map $F$. This noninvertibility allows the preimages of the period-12 saddle cycle to serve as points of separation between the two directions of the manifolds. The complete basin of attraction for the period- 12 node consists of these immediate disc-formed domains together with all their preimages in the main tongues as well as in their preimages. A similar basin structure was found in Ref. [29] for a twodimensional, noninvertible map derived from a problem in radiophysics.

The next transformations of the phase portrait to occur involve a transcritical 1:3 subharmonic bifurcation [28] of the period-4 focus at $\varepsilon \cong-1.0581$, and a Hopf bifurcation of the stable period- 12 solution at $\varepsilon \cong-1.0562$. In the first of these bifurcations the points of the period-12 saddle cycle pass right through the points of the period- 4 focus and destabilize the latter cycle. Figure 14 illustrates the basins of attraction that exist after this bifurcation. Here $\varepsilon$ 


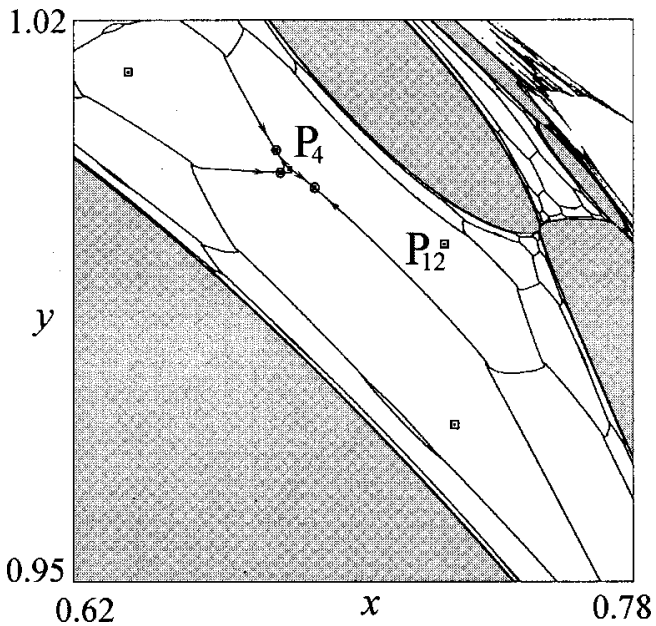

FIG. 14. A transcritical 1:3 subharmonic bifurcation has transformed the stable period- 4 focus into an unstable focus. The small circles are points of the period-12 saddle that have passed right through the period- 4 focus. Here $\varepsilon=-1.0564$.

$=-1.0564$, and the points of the period-12 saddle are again shown as small circles. The period-12 focus is now the only attracting set inside the tongues that emanate from the synchronous period-2 cycle. The Hopf bifurcation at $\varepsilon$ $=-1.0581$ produces a period- 12 invariant circle (referred to as a torus) $T_{12}$ corresponding initially (with high probability) to quasiperiodic motion. With a further increase of the coupling parameter, the dynamics becomes noninvertible and the torus breaks down [30], producing either regular periodic solutions or chaos in the form of a so-called Cantor torus. In both cases we can have either a single stable solution or two coexisting and mutually symmetric attractors. For $\varepsilon$ $\in[-1.054,-1.053]$, for instance, the dynamics is reduced to two symmetrically located stable period- 12 solutions and two saddle cycles of the same period.

Remark: This type of organization appears to be characteristic for coupled symmetrical map systems. In a detailed study of bifurcation phenomena in systems of diffusively coupled logistic maps, Giberti and Vernia [31] found that the presence of closed invariant manifolds containing periodic orbits can play a prominent role for the dynamics. Typically, for an $N$-dimensional system, such a manifold will hold up to $N$ stable node solutions and $N$ saddles. In the direction normal to the manifold the attraction can be relatively strong so that trajectories rapidly settle onto the closed invariant curve. Along the manifold, on the other hand, the action of the map can be nearly indistinguishable from the identity transformation, and the dynamics may be very slow. Hence, one can observe extremely long transients.

For other values of the coupling parameter, the dynamics on the Cantor torus produced through the breakdown of $T_{12}$ involves two coexisting chaotic attractors. For $\varepsilon$ $=-1.0517$, for instance, two $(5 \times 12)$-piece chaotic attractors exist. At $\varepsilon \cong-1.05134$, a single 12 -piece chaotic attractor is born and for slightly higher values of the coupling parameter, the 12-piece attractor merges into a four-piece attractor. This last process gives rise to the appearance of so-called rare points [32], indicating that merging takes place across a fractal basin boundary. Finally, at $\varepsilon \cong-1.0458$ the chaotic attractor and its basin of attraction disappear in a

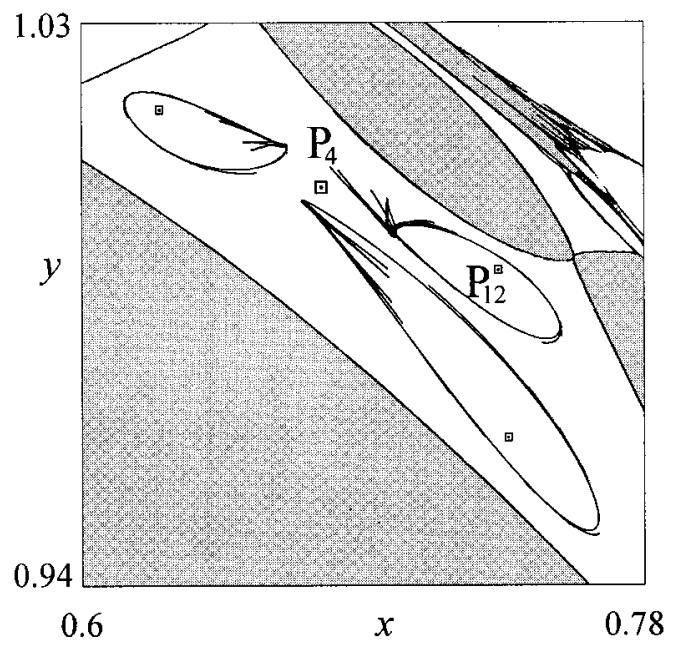

FIG. 15. For $\varepsilon=-1.0513$, a single 12-piece chaotic attractor exists in the repelling tongues emanating from the symmetric period-2 repeller. As the coupling parameter is further increased, the 12-piece chaotic attractor merges into a four-piece attractor to finally disappear together with its basin of attraction in a boundary crisis.

boundary crises, leaving only a chaotic saddle of period 4 in the region around the original asynchronous period-4 cycle. Figure 15 shows a phase portrait for $\varepsilon=-1.0513$. For this value of the control parameter, our coupled map system $F$ displays a 12-piece chaotic attractor situated in the repelling tongues issued from the points of the symmetric period-2 cycles. As described above, this attractor has been produced via breakdown (loss of differentiability) of the 12-piece torus $T_{12}$. Also shown in the figure are the locations of the period-4 and -12 cycles, both of which are now unstable focuses.

This completes our discussion of the complex scenario that unfolds after the supercritical transverse destabilization of the symmetric period- 2 cycle. After the asynchronous chaotic attractor has disappeared in a boundary crisis, no other attracting set is observed inside the larger absorbing area, and the basin of attraction for the synchronized chaotic state remains locally riddled until, at $\varepsilon \cong-1.0385$ a blowout bifurcation takes place. In this bifurcation the typical transverse Lyapunov exponent for trajectories on $A$ becomes positive, and the synchronized chaotic state loses its average attraction. However, the larger absorbing area $\mathcal{A}$ still exists inside the basin of attraction, and, as described in Ref. [16], the blowout bifurcation leads to on-off intermittency. The chaotic attractor spreads over the whole area of the absorbing set. However, trajectories starting near the synchronization manifold cannot diverge to infinity. For slightly higher values of the coupling parameter, the two-dimensional chaotic attractor undergoes an inverse boundary crisis by which it decomposes into two mutually symmetric chaotic attractors. For $\varepsilon=-1$, these attractors are both restricted to onedimensional manifolds, and one can observe the phenomenon of intermingled basins of attraction [16]. Each attractor here has a basin that is riddled with initial conditions that lead to the other attractor. The mutually symmetric chaotic attractors finally disappear in a boundary crisis at $\varepsilon$ $\cong-0.935$. When this occurs, the larger absorbing area in 


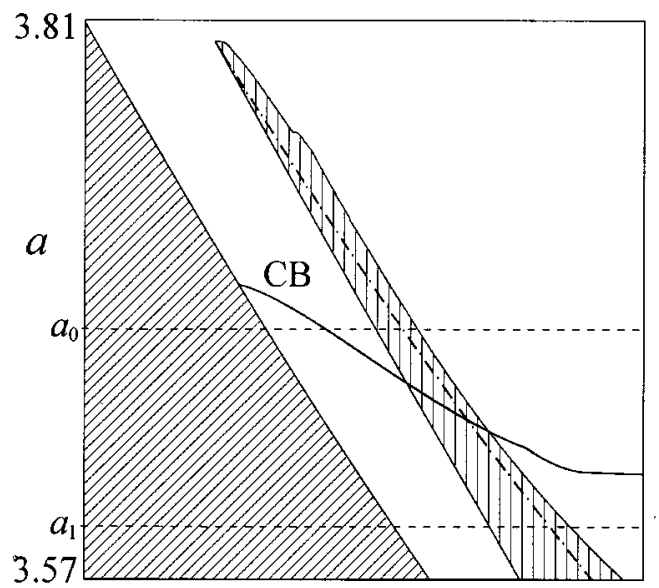

(a) -1.35

$\varepsilon$

$-1.0$

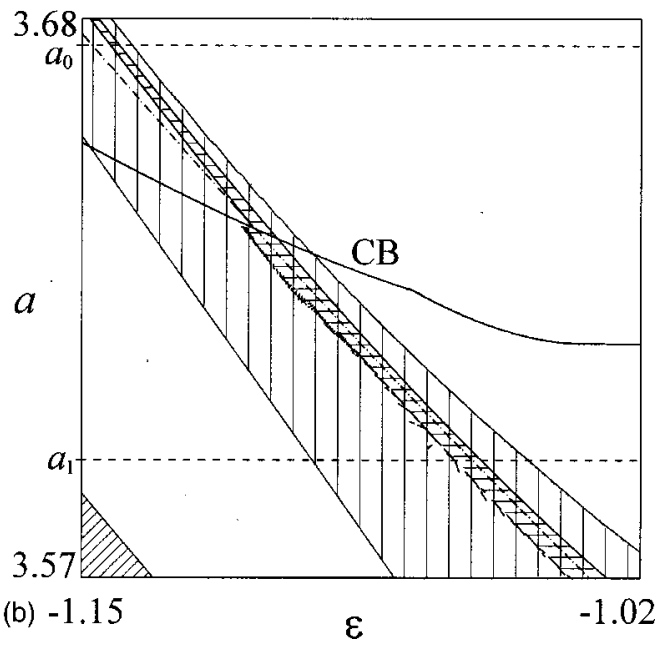

FIG. 16. (a) Phase diagram illustrating the region (vertically crosshatched) in which attracting states exist inside the repelling tongues emanating from the symmetric period- 2 cycle. The triangular region to the left is the region where the symmetric period-2 cycle is transversely stable (compare with Fig. 1). (b) Magnification illustrating the region of stability for the period-12 cycle (horizontally crosshatched). The curve marked CB represents the contact bifurcation.

Fig. 11 has made contact with the basin boundary and, hence, has ceased to exist.

The phase diagram in Fig. 16(a) gives an overview of the main bifurcations involved in the above scenarios, and clarifies the difference between the scenarios observed for $a$ $=a_{0}$ and for $a=a_{1}$. In this diagram the crosshatched region to the left represents parameter values for which the symmetric period-2 cycle is transversely stable (compare with Fig. 1 ). At the right hand edge of this region, the period-2 cycle undergoes a supercritical transverse period doubling, producing a period- 4 saddle cycle outside the main diagonal. The vertically crosshatched finger to the right of this bifurcation curve represents the regions of parameter space in which attracting states exist in the repelling tongues emanating from the points of the period-2 repeller. The left hand edge of this finger is the bifurcation curve in which the asymmetric period-4 saddle stabilizes in an inverse, subcritical period doubling. The broken curve running through the middle of the finger is the Hopf bifurcation curve for the period-4 cycle, and the right hand edge of the finger represents the boundary crisis in which a four-piece chaotic attractor disappears through collision with its basin boundary.

The curve marked CB represents the contact bifurcation between the absorbing area and the basin boundary for the synchronized chaotic state $A$. For $a=a_{0}$, as the coupling parameter is increased, the contact bifurcation occurs before the stabilization of the asynchronous period-4 cycle. This leads to the scenario that we described in Sec. III. Here the transition from local to global riddling of the basin of attraction for $A$ takes place via the contact bifurcation. For $a$ $=a_{1}$, on the other hand, the asymmetric period- 4 cycle stabilizes before the contact bifurcation, and the scenario described in the present section takes place. If the nonlinearity parameter is precisely equal to $a_{1}$, we do not observe a transition to global riddling. For a value of $a$ slightly larger than $a_{1}$, however, we observe a transition to the peculiar form of global riddling that manifests itself in the form of randomly scattered dots within the region otherwise assigned as the basin for $A$ [27].

Figure 16(b) provides a little more detail about the bifurcation structure. Again, the vertically crosshatched region represents parameter values for which stable solutions exist inside the repelling tongues issued from the symmetric period-2 cycle. The left edge is the bifurcation curve in which the asynchronous period- 4 cycle stabilizes, and the right edge represents the boundary crisis where the repelling tongues disappear. The narrower, horizontally crosshatched area is the region where the stable period-12 cycle exists. This cycle arises in a subharmonic saddle-node bifurcation to be destabilized at higher values of the coupling parameter in a Hopf bifurcation leading to the above mentioned 12-piece torus $T_{12}$. The punctuated curve running through the middle of the finger again represents the bifurcations in which the asynchronous period- 4 cycle loses its stability. Above $a$ $\cong 3.64$, where the period- 12 cycle has not yet appeared, this happens via a Hopf bifurcation. For $a<3.64$, however, the period-4 cycle loses its stability in a 1:3 subharmonic transcritical bifurcation.

At the other end of the interval of asymptotic stability for $a=a_{1}$, the transverse destabilization takes place in the form of a subcritical pitchfork bifurcation of the symmetric period-2 cycle at $\varepsilon \cong-1.46$. This leads directly to a globally riddled basin of attraction [16]. Let us finally note that, for $a=a_{1}$, there is an additional interval of weak stability for the synchronized chaotic state around $\varepsilon=0.3$. However, this interval does not contain regions of asymptotic stability for the chaotic set. Neither is there an absorbing area. Hence the basin of attraction is globally riddled in the whole interval.

\section{CONCLUSION}

One of the main problems relating to the application of chaos synchronization methods concerns the behavior of the coupled system once the synchronization breaks down. In certain situations, one can observe an abrupt transition to a state in which the trajectories can diverge far out in phase space. In other situations the dynamics shows minor and smoothly appearing bursts away from the synchronized chaotic state. Considering two coupled identical, onedimensional maps, we have derived exact analytical conditions for the transverse destabilization of the period- 2 cycle 
to be either subcritical or supercritical. In Ref. [21] we provided the results for the generic case of an $n$-periodic cycle.

Based on these results, we conjecture that the riddling bifurcation will be hard or soft depending on the subcritical or supercritical nature of the first transverse destabilization of a period cycle. Immediately after a supercritical transverse bifurcation, the unstable manifolds of the emerged saddle cycle(s) will stretch along the synchronization manifold. Together with segments of critical curves, segments of these manifolds will define a closed invariant region (the mixed absorbing area) from which trajectories cannot escape. Following a subcritical transverse bifurcation, on the other hand, no such mixed absorbing area will exist after the bifurcation, and direct access may be created for the trajectories to move far away.

Our analysis is based on the assumption that the first orbit to lose its transverse stability is a cycle. As shown by Hunt and Ott [33], this is the generic situation. In their discussion of orbits with optimal properties, they conjectured that for a typical one-dimensional map, nonperiodic orbits with optimal properties occur on a set of zero Lebesgue measure in parameter space. Moreover, the measure of parameter values for which the optimal orbit has a period greater than some interger $N$ decreases exponentially with $N$. (The optimal property of significance for our analysis is a transverse eigenvalue that exceeds unity.)

We have also described two different scenarios by which global riddling of the basin of attraction can arise after an initial supercritical riddling bifurcation. This analysis has clearly emphasized the important roles played by the concepts of absorbing and mixed absorbing areas developed mainly by Mira et al. [19] and applied and extended by Bischi, Stefanini, and Gardini [20]. In the first scenario the transition from locally to globally riddled basins of attraction occurs in a contact bifurcation as a repelling orbit situated at the boundary of the basin of attraction crosses the common boundaries for the absorbing and the mixed absorbing areas. In the second scenario the transition to the globally riddled basin of attraction (for $a>a_{1}$ ) occurs via a secondary local bifurcation. This bifurcation stabilizes the saddle cycle produced in the soft riddling bifurcation, and hence creates a new attracting state inside the absorbing area. It is worth noticing that the characters of the globally riddled basins of attraction generated by these two scenarios are quite different.

Let us finally note that although we have considered a simple system of only two coupled logistic maps, our model is directly related to the problem of clustering in globally coupled map systems as described by Kaneko [7]. To illus- trate this relation let us consider the $K$-dimensional coupled map system

$$
x_{n+1}(i)=f\left(x_{n}(i)\right)+\frac{2 \varepsilon}{K} \sum_{j=1}^{K}\left\{f\left(x_{n}(j)\right)-f\left(x_{n}(i)\right)\right\},
$$

where $\varepsilon$ is the coupling constant and $f(x)=a x(1-x)$ the one-dimensional logistic map. $n=1,2, \ldots$ denotes the discrete time variable, and $i, j=1,2, \ldots, K$ is a number associated with the individual map. $a$ is assumed to be given a value such that the dynamics of $f(x)$ is chaotic. As shown by Kaneko [7], between the turbulent states at low values of the coupling parameter and the fully synchronized (or coherent) state at high values of $\varepsilon$, there is a coupling interval where the asymptotic behavior of (6.1) is dominated by two-cluster dynamics. Under these conditions we can make the identifications

$$
\begin{gathered}
x_{n} \equiv x_{n}(1)=\cdots=x_{n}\left(K_{1}\right), \\
y_{n} \equiv x_{n}\left(K_{1}+1\right)=\cdots=x_{n}(K),
\end{gathered}
$$

where $K_{1}\left(1 \leqslant K_{1}<K\right)$ is the number of oscillators in one of the clusters. It is now easy to see that the variables $x$ and $y$ satisfy the relations

$$
\begin{gathered}
x_{n+1}=f\left(x_{n}\right)+2 \varepsilon \frac{K-K_{1}}{K}\left(f\left(y_{n}\right)-f\left(x_{n}\right)\right), \\
y_{n+1}=f\left(y_{n}\right)+2 \varepsilon \frac{K_{1}}{K}\left(f\left(x_{n}\right)-f\left(y_{n}\right)\right) .
\end{gathered}
$$

If, finally, $K_{1}=K / 2$, system (6.3) reduces to a system of two symmetrically coupled logistic maps with nonlinear coupling. Hence we conclude that the transition from one- to two-cluster behavior in the $K$-dimensional, globally coupled map system will follow the same general scenarios as we described above.

Particularly when the chaotic dynamics of the individual map is highly developed (i.e., for large values of the nonlinearity parameter $a$ ), instead of two-cluster dynamics one finds a glassy phase where a couple of large clusters coexist with many small clusters [7]. From a thermodynamic point of view the glassy phase may be interpreted as a frozen disequilibrium structure similar to the frozen states that one can observe in spin glass models. From the point of view of our more detailed bifurcation analysis, however, the glassy phase arises from the existence, together with the synchronized chaotic state, of stable asynchronous solutions that each commands a certain basin of attraction.
[1] E. Mosekilde, Topics in Nonlinear Dynamics, Applications to Physics, Biology and Economic Systems (World Scientific, Singapore, 1996); Chaotic Oscillators, Theory and Applications, edited by T. Kapitaniak (World Scientific, Singapore, 1992).

[2] H. Bohr, K. S. Jensen, T. Petersen, B. Rathjen, E. Mosekilde, and N.-H. Holstein-Rathlou, Parallel Comput. 12, 113 (1989);
J. Halloy, Y. X. Li, J. L. Martiel, B. Wurster, and A. Goldbeter, Phys. Lett. A 151, 33 (1990); K. Kaneko, Physica D 75, 55 (1994); M. I. Rabinovich, H. D. I. Abarbanel, R. Huerta, R. Elson, and A. I. Selverston, IEEE Trans. Circuits Syst., I: Fundam. Theory Appl. 44, 997 (1997).

[3] V. S. Anishchenko, T. E. Vadivasova, D. E. Postnov, and M. A. Safonova, Int. J. Bifurcation Chaos Appl. Sci. Eng. 2, 633 
(1992); V. S. Anishchenko, T. E. Vadivasova, D. E. Postnov, O. V. Sosnovtseva, L. O. Chua, and C. W. Wu, ibid. 5, 1525 (1995).

[4] M. G. Rosenblum, A. S. Pikovsky, and J. Kurths, Phys. Rev. Lett. 76, 1804 (1996); G. V. Osipov, A. S. Pikovsky, M. G. Rosenblum, and J. Kurths, Phys. Rev. E 55, 2353 (1997).

[5] H. Fujisaka and T. Yamada, Prog. Theor. Phys. 69, 32 (1983); 75, 1087 (1986); L. M. Pecora and T. L. Carroll, Phys. Rev. Lett. 64, 821 (1990); Phys. Rev. A 44, 2374 (1991).

[6] M. G. Rosenblum, A. S. Pikovski, and J. Kurths, Phys. Rev. Lett. 78, 4193 (1997).

[7] K. Kaneko, Physica D 41, 137 (1990); 75, 55 (1994).

[8] K. Pyragas, Phys. Lett. A 181, 203 (1993); Phys. Rev. E 54, R4508 (1996); M. Vieira and A. J. Lichtenberg, ibid. 56, R3741 (1997).

[9] N. F. Rulkov, Chaos 6, 262 (1996); G. Kolumbán, M. P. Kennedy, and L. O. Chua, IEEE Trans. Circuits Syst., I: Fundam. Theory Appl. 44, 927 (1997).

[10] J. C. Alexander, J. A. Yorke, Z. You, and I. Kan, Int. J. Bifurcation Chaos Appl. Sci. Eng. 2, 795 (1992); J. C. Alexander, B. R. Hunt, I. Kan, and J. A. Yorke, Ergod. Th. Dyn. Syst. 16, 651 (1996).

[11] J. C. Sommerer and E. Ott, Nature (London) 365, 136 (1993); E. Ott and J. C. Sommerer, Phys. Lett. A 188, 39 (1994).

[12] P. Ashwin, J. Buescu, and I. Stewart, Phys. Lett. A 193, 126 (1994); Nonlinearity 9, 703 (1996); J. F. Heagy, T. L. Carroll, and L. M. Pecora, Phys. Rev. E 52, R1253 (1995).

[13] L. Yu, E. Ott, and Q. Chen, Physica D 53, 102 (1992).

[14] N. Platt, E. A. Spiegel, and C. Tresser, Phys. Rev. Lett. 70, 279 (1993).

[15] Y.-C. Lai, C. Grebogi, J. A. Yorke, and S. C. Venkataramani, Phys. Rev. Lett. 77, 55 (1996).

[16] Yu. L. Maistrenko, V. L. Maistrenko, A. Popovich, and E. Mosekilde, Phys. Rev. E 57, 2713 (1998); Phys. Rev. Lett. 80, 1638 (1998).

[17] Yu. Maistrenko, T. Kapitaniak, and P. Szuminski, Phys. Rev.
E 56, 6393 (1997); T. Kapitaniak, Yu. Maistrenko, A. Stefanski, and J. Brindley, ibid. 58, R6253 (1998).

[18] S. C. Venkataramani, B. R. Hunt, E. Ott, D. J. Gauthier, and J. C. Bienfang, Phys. Rev. Lett. 77, 5361 (1996); S. C. Vankataramani, B. R. Hunt, and E. Ott, Phys. Rev. E 54, 1346 (1996).

[19] C. Mira, L. Gardini, A. Barugola, and J.-C. Cathala, Chaotic Dynamics in Two-Dimensional Noninvertible Maps (World Scientific, Singapore, 1996); R. H. Abraham, L. Gardini, and C. Mira, Chaos in Discrete Dynamical Systems (SpringerVerlag, Berlin, 1997).

[20] G.-I. Bischi, L. Stefanini, and L. Gardini, Mater. Comput. Sim. 44, 559 (1998).

[21] Yu. L. Maistrenko, V. L. Maistrenko, O. Popovych, and E. Mosekilde (unpublished).

[22] G.-I. Bischi and L. Gardini, Phys. Rev. E 58, 5710 (1998).

[23] I. U. Bronstein and A. Ya. Kopanskii, Smooth Invariant Manifolds and Normal Forms (World Scientific, Singapore, 1996).

[24] C. Mira, C. Rauzy, Yu. Maistrenko, and I. Sushko, Int. J. Bifurcation Chaos Appl. Sci. Eng. 6, 2299 (1996).

[25] S. E. Newhouse, Topology 13, 9 (1974).

[26] S. V. Gonchenko, L. P. Shil'nikov, and D. V. Turaev, Chaos 6, 15 (1996)

[27] V. Astakhov, A. Shabunin, T. Kapitaniak, and V. Anishchenko, Phys. Rev. Lett. 79, 1014 (1997).

[28] G. Iooss and D. D. Joseph, Elementary Stability and Bifurcation Theory, 2nd ed. (Springer-Verlag, Berlin, 1980).

[29] V. Maistrenko, Yu. Maistrenko, and I. Sushko, Int. J. Bifurcation Chaos Appl. Sci. Eng. 2, 383 (1994).

[30] Dynamical Systems V, edited by V. I. Arnol'd, Encyclopedia of Mathematical Sciences (Springer-Verlag, Berlin, 1994).

[31] C. Giberti and C. Vernia, Int. J. Bifurcation Chaos Appl. Sci. Eng. 3, 1503 (1993), Chaos 4, 651 (1994).

[32] Yu. Maistrenko, I. Sushko and L. Gardini, Chaos Solitons Fractals 9, 1373 (1998).

[33] B. R. Hunt and E. Ott, Phys. Rev. Lett. 76, 2254 (1996); S. M. Zoldi and H. S. Greenside, ibid. 80, 1790 (1998). 